\title{
Black Hole Simulations in Condensed Matter Systems
}

\author{
Luiz Gustavo Egito da Silva*, ${ }^{a}$ Antônio de Pádua Santos ${ }^{a}$, and Erms Pereira ${ }^{a}$ \\ ${ }^{a}$ Departmento de Física \\ Universidade Federal Rural de Pernambuco UFRPE \\ http://www.ufrpe.br/ \\ E-mail: egito80@yahoo.com.br,antonio.padua@ufrpe.br, \\ erms.pereira@ufrpe.br
}

\begin{abstract}
Black holes have always been of great interest in gravitation and high energy particles. Through their interaction with light and matter, it is possible to understand their behavior. However, there is an apparent difficulty in studying it in the laboratory, in addition to the complex equations representing them, in particular when we left for black holes without singularities. In this work, we use a finite element software to simulate the metric of black holes without singularities in condensed matter systems. For these non-singular black holes, the idea is to use the metric tensor as a unitary element $d s^{2}$ and make this metric as the electric permittivity of material to be simulated in software. We study a plane wave on the influence of the black hole, presenting how the light is disturbed by the presence of the black hole. Then it is possible to identify a light signature of the presence of black holes, thus helping their detection and study of their properties .
\end{abstract}

International Conference on Black Holes as Cosmic Batteries: UHECRs and Multimessenger Astronomy BHCB2018

12-15 September, 2018

Foz do IguaÃ $\breve{g} u$, Brasil

${ }^{*}$ Speaker. 


\section{Introduction}

The condensed matter usually consists of a wide range of problems in systems that may or may not be in the perfect solid state, essentially we have to things like (solid phases, liquid phase, superconducting phase, ferromagnetic phase, etc.) [1]. Moreover, we will have materials that have unique macroscopic properties, that is, are material designed to have a property that is not found in natural materials. Furthermore, due to greater scale repetition have recently been called metamaterials [2]. But depending on what you are trying to accomplish, each approach has a different set of tools, in our case we use the general relativity in condensed matter or engineered material may seem surprising: relativity has been associated with the physics of gravitation and cosmology. Furthermore, we know that black holes have always been of great interest in gravitation and high energy particles, due to the singularity surrounding such objects in practical terms, the singularity means failure, a "fissure" in the equations and solutions of general relativity, that is, a dimensionless point and infinite mass and energy [3]. Already the non-singular black holes are entities where singularities have been removed by regularization, that is, non-divergence or non-occurrence of singularity, and is satisfied only in the case de Sitter's space-time [4]. The de Sitter's space-time is a more fundamental quotient space than the Einstein equation by the addition of the cosmological term and it can be defined as a sub manifold of a Minkowski space of a higher dimension and can be constructed independently of any gravitational theory [5]. However, the available software package is not user-friendly [6], making the study difficult. In this work, we use the finite element software COMSOL Multiphysics ${ }^{\circledR}{ }^{\circledR}$ [7]to model the propagation of light around non-singular black holes using a material with special dielectric properties that present the identical effective metric, according to the work [8].

\section{Theory and Description}

Our work aims at a special class of the interaction of the black holes through their interaction with light and matter, these can also be characterized by regular black holes, non-singular black holes or Bardeen black holes [9]. In addition, the multiphysics modeling presented in this work tried to describe artificially produced structures common to metamaterials, where these nonsingular metrics are made using in magnetic metamaterials. Now, we will introduce to these nonsingular black holes a metric tensor unitary element cofactor $d s^{2}$, his representing the Bardeen metric [10, 11, 12]:

$$
d s^{2}=-f(r) d t^{2}+\frac{d r^{2}}{f(r)}+r^{2}\left(d \theta^{2}+\sin ^{2} \theta d \phi^{2}\right) .
$$

The Bardeen's solution is a result of the gravitational field of Einstein in the presence of an electromagnetic field, the well-known Reissner-Nordström solution. Studying light trajectory in the geometry of a black hole type regular we have the following expression for $f(r)$ in Bardeen black hole. [9]:

$$
f(r)=1-\frac{2 m(r)}{r}
$$

and

$$
m(r)=\frac{M r^{3}}{\left(r^{2}+e^{2}\right)^{2}}
$$


where $M$ and $e$ are constants.

In the laboratory, it is theoretically possible to simulate metric using condensed matter systems $[13,14]$, and non-singular metrics can be effectively constructed via concentric layers [15] of subwavelength unit structures. With this construction, one can control, for example, the radial variation of the dielectric properties. A way to simulate the propagation of light in a metric with components $g_{\alpha \beta}$ is to consider the light propagating in a material with the following dielectric permittivity [8]:

$$
\varepsilon^{i j}=-\frac{\sqrt{-g}}{\sqrt{\gamma}} \frac{g^{i j}}{g_{00}},
$$

where $g=\operatorname{det}\left(g_{\alpha \beta}\right), \gamma=\operatorname{det}\left(\gamma_{i j}\right)$, and $\gamma_{i j}$ are the components of the flat spatial metric.

\section{Results and Discussion}

Using the metric of line element Eq. (2.1) with the terms (2.2) and (2.3) to determine the effective components of the dielectric tensor $\varepsilon^{i j}$ using Eq. (2.4), we implemented simulations using the finite element software COMSOL Multiphysics. This software solves the Maxwell equation for a given dielectric tensor, allowing one to use it to model the propagation of light close to a nonsingular black hole.

Using Gaussian waves to model laser beams and plane waves, both with wavelength $\lambda=5 \mu \mathrm{m}$, in the flat and non-singular black hole (with $M=1$ and $e=2$ ) metrics, we found the results shown in Fig. 1, for a $20 \mu \mathrm{m}$-square region adequate to the condensed matter simulation. In Figs. 1(a)-(b), one notices a spread of the Gaussian beam and a deflection in the direction of square's center, the location of the non-singular black hole, resulted from the different radial distance from portions of the ray to the center of the black hole. Such phenomena repeat for the plane wave, as one can see in Figs. 1(c) and (d). Beyond the fringe phenomenon due to a boundary effect, we noticed a split on the plane wave after the non-singular black hole. Again because the different radial distance from portions of the ray to the center of the black hole, this split can be used as optical signatures of such non-singular black holes. These results are similar to experimental realizations for photonic black holes [16].

\section{Conclusions}

In this work, we simulated the propagation of plane and Gaussian light waves in a solid material with dielectric properties that mimic the metric of a non-singular black hole. We found a spread and split of the incident beam, due to the interaction of the black hole on portions of the beam with different radial distances. Our results are similar to experimental achievement in photonic black holes [16] and can be used to identify non-singular black holes using light scattering.

\section{Acknowledgement}

We want to express our thanks to the Instituto Serrapilheira, the National Council for Scientific and Technological Development (CNPq - 465259/2014-6), the Coordination for the Improvement of Higher Education Personnel (CAPES), the National Institute of Science and Technology Complex Fluids (INCT-FCx), and the São Paulo Research Foundation (FAPESP - 2014/50983-3). 


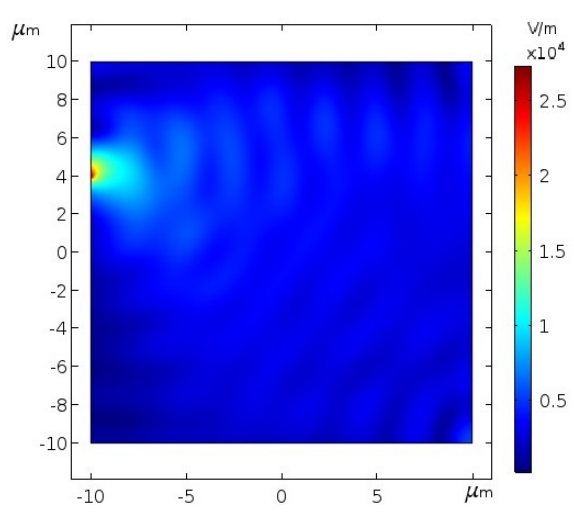

(a) Gaussian beam / flat spacetime

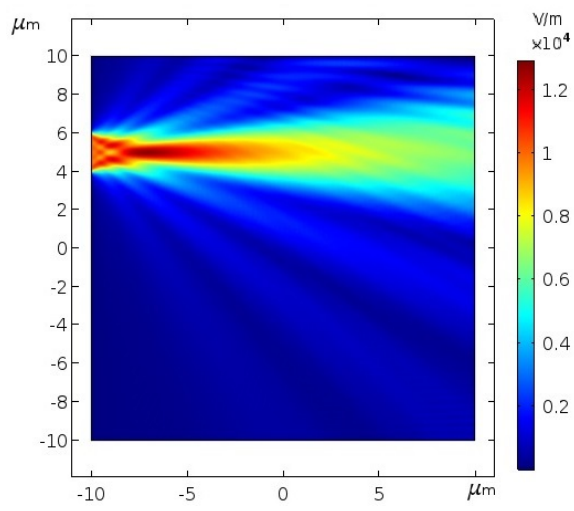

(c) Plane wave / flat spacetime

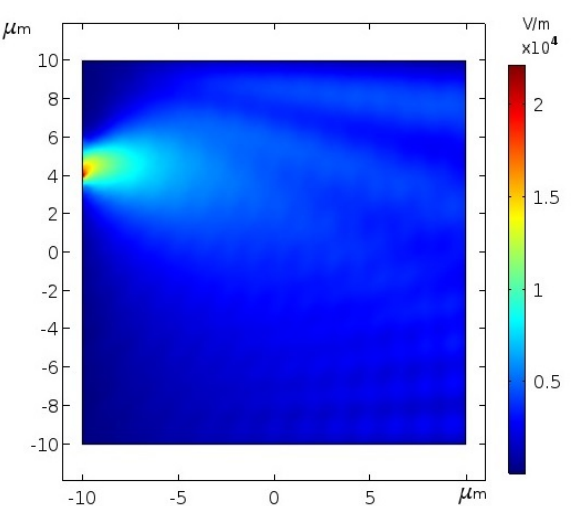

(b) Gaussian beam / nonsingular black hole

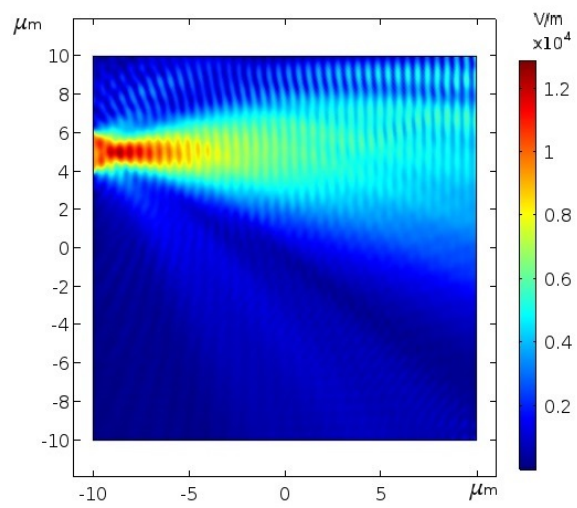

(d) Plane wave / nonsingular black hole

Figure 1: Propagation of light in a material simulating a flat spacetime, (a) and (c), and a nonsingular black hole, (b) and (d). The parameters used in this simulation were wavelength $\lambda=5 \mu \mathrm{m}$, $M=1$ and $e=2$.

\section{References}

[1] W. Kohn, An essay on condensed matter physics in the twentieth century, Reviews of Modern Physics 71 (1999) S59.

[2] H. Chen, C. Chan, P. S. N. Materials and U. 2010, Transformation optics and metamaterials, nature.com (2010) .

[3] S. M. Carroll, Spacetime and geometry. An introduction to general relativity. Pearson Education Limited, 2004.

[4] T. Hartman, Lecture Notes on Classical de Sitter Space, Cornell University (2017).

[5] U. Moschella, The de Sitter and anti-de Sitter sightseeing tour, in Einstein, 1905-2005, pp. 120-133, Springer, (2006).

[6] W. Christian, M. Belloni and A. Cox, General Relativity (GR) Package, 2008.

[7] COMSOL AB, COMSOL Multiphysics $®, 2015$. 
[8] U. Leonhardt and T. G. Philbin, General relativity in electrical engineering, New Journal of Physics 8 (2006) 247.

[9] J. M. Bardeen, Non-singular general-relativistic gravitational collapse, in Proc. Int. Conf. GR5, Tbilisi, vol. 174, 1968.

[10] R. Carballo-Rubio, F. Di Filippo, S. Liberati, C. Pacilio and M. Visser, On the viability of regular black holes, Journal of High Energy Physics 2018 (2018) 23.

[11] J. Neves, Well-behaved relativity: regular black holes, Revista Brasileira de Ensino de Fl'isica 39 (2017) .

[12] R. Carballo-Rubio, F. Di Filippo, S. Liberati and M. Visser, Phenomenological aspects of black holes beyond general relativity, Physical Review D 98 (2018) 124009.

[13] E. R. Pereira and F. Moraes, Flowing liquid crystal simulating the schwarzschild metric, Cent. Eur. J. Phys. 9 (2011) 1100.

[14] A. de Padua, F. Parisio-Filho and F. Moraes, Geodesics around line defects in elastic solids, Phys. Lett. A 238 (1998) 153.

[15] Q. Cheng, T. J. Cui, W. X. Jiang and B. G. Cai, An electromagnetic black hole made of metamaterials, arXiv preprint arXiv:0910.2159 (2009).

[16] D. A. Genov, S. Zhang and X. Zhang, Mimicking celestial mechanics in metamaterials, Nature Physics 5 (2009) 687. 